\title{
Performance of ESPRIT for estimating mixtures of complex exponentials modulated by polynomials
}

\author{
Roland Badeau, Member, IEEE, Gaël Richard, Senior Member, IEEE, and Bertrand David, Member, IEEE
}

\begin{abstract}
High Resolution (HR) methods are known to provide accurate frequency estimates for discrete spectra. The Polynomial Amplitude Complex Exponentials (PACE) model, also called quasipolynomial model in the literature, was presented as the most general model tractable by HR methods. A subspacebased estimation scheme was recently proposed, derived from the classical ESPRIT algorithm. In this paper, we focus on the performance of this estimator. We first present some asymptotic expansions of the estimated parameters, obtained at the first order under the assumption of a high signal-to-noise ratio. Then the performance of the generalized ESPRIT algorithm for estimating the parameters of this model is analyzed in terms of bias and variance, and compared to the Cramér-Rao bounds. This performance is studied in an asymptotic context, and it is proved that the efficiency of undamped single poles estimators is close to the optimality. Moreover, our results show that the best performance is obtained for a proper dimensioning of the data. To illustrate the practical capabilities of the generalized ESPRIT algorithm, we finally propose an application to ARMA filter synthesis, in the context of system conversion from continuous time to discrete time.
\end{abstract}

Index Terms-ESPRIT, high resolution (HR), multiple eigenvalues, performance analysis, perturbation theory, polynomial modulation.

\section{INTRODUCTION}

$\mathbf{H}$ IGH Resolution (HR) methods, such as the well-known ESPRIT algorithm [1], are very classical techniques for estimating mixtures of complex exponentials in white noise. However the underlying Exponential Sinusoidal Model (ESM), although the most studied in the literature, is not the most general model tractable by HR methods. Indeed the ESM only accounts for systems with single poles, whereas one can find examples of systems involving multiple poles, which generate mixtures of complex exponentials modulated by polynomials, as shown in [2]. For instance, critically damped harmonic oscillators involve a double pole [3]. Laguerre functions are a special case of signals with multiple poles (the exponentials are modulated by Laguerre polynomials), often used in the estimation of time delays [4], [5], and in biomedical engineering, for modeling fluorescence decay [6]. Signals with multiple poles also appear in quantum physics, as solutions of the Schrödinger equation for hydrogen-like atoms [7], in

Roland Badeau, Gaël Richard and Bertrand David are with the Department of Signal and Image Processing, École Nationale Supérieure des Télécommunications (ENST), Paris, France. E-mail: [roland.badeau, bertrand.david, gael.richard]@enst.fr.

(C) 2007 IEEE. Personal use of this material is permitted. However, permission to reprint/republish this material for advertising or promotional purposes or for creating new collective works for resale or redistribution to servers or lists, or to reuse any copyrighted component of this work in other works must be obtained from the IEEE. laser physics, as transverse laser modes [8], and in finance, for modeling the evolution of interest rates [9].

In order to estimate the parameters of this more general Polynomial Amplitude Complex Exponentials (PACE) model, also referred to as the quasipolynomial model [10] in the literature, we proposed in [2] a generalization of the ESPRIT algorithm for estimating multiple poles. The polynomial amplitude parameters can then be recovered by means of a Least Squares (LS) method. The performance of an estimator is generally described in terms of bias and variance, the latter being generally compared to the Cramér-Rao bound (CRB), in terms of statistical efficiency [11]. An analysis of the Cramér-Rao bounds for the frequencies and damping factors of complex quasipolynomials in white noise was proposed in [10]. In [12], we derive analytic expressions of the Cramér-Rao bounds for the frequencies, damping factors, amplitudes and phases of quasipolynomials in colored noise, and these expressions are simplified in an asymptotic context. In particular, it is shown that the CRB for the parameters associated to a multiple pole present an exponential increase with the order of the pole, which suggests that the practical estimation of the PACE model is only possible if the exponentials are modulated by polynomials of low order.

Unfortunately, in the case of HR methods, the bias and variance cannot be calculated analytically, because the extraction of polynomial roots, or matrix eigenvalues, induces a complex relationship between the statistics of the signal and those of the estimators. In the case of the ESM however, asymptotic results were obtained with the perturbation theory. These results rely either on the hypothesis of a high window length $(N \rightarrow+\infty$, in the case where all the poles are on the unit circle), or on the hypothesis of a high signal-to-noise ratio $(\mathrm{SNR} \rightarrow+\infty)$. For instance, it was established in [13], [14] that the Prony [15] and Pisarenko [16] methods are very inefficient: their variances are much greater than the Cramér-Rao bounds. Conversely, the Minimum Norm method [17], MUSIC [18], ESPRIT [1] and Matrix Pencil [19] have an asymptotic efficiency close to 1 , as shown in [20]-[24]. More precisely, it was proved in [22], [23] in the case of undamped sinusoids that MUSIC and ESPRIT perform similarly, with a slight advantage for ESPRIT. This was confirmed in [24] in the more general case of the ESM: ESPRIT and Matrix Pencil are less sensitive to noise than MUSIC.

In the case of the PACE model, it was shown in [2] that the presence of noise scatters the multiple poles into several single poles, forming the vertices of a regular polygon as a first order approximation. However the original multiple pole can be recovered by computing the arithmetic mean of the scattered poles. Below, we analyze the performance of this approach in 
presence of colored noise and under the high SNR hypothesis, in terms of first order perturbations. These developments are utilized to show that the estimators proposed in [2] for the PACE model are unbiased, and their variances are calculated and compared to the Cramér-Rao bounds. Additionally, by considering a high observation length and a white noise, it is shown that the efficiency of the estimators is close to 1 . In particular, we generalize a result presented in [19], which provides the ideal dimensioning of the data matrix in order to improve the efficiency of single poles estimators. However, our simulation results confirm that the practical estimation of the parameters is only possible for poles of low order. To illustrate the capabilities of our estimation method, we finally propose an application to ARMA filter synthesis, in the context of system conversion from continuous time to discrete time.

The paper is organized as follows. Section II describes the PACE model and the estimation method introduced in [2]. Then the influence of an additive perturbation onto the estimated frequencies, damping factors, amplitudes and phases is studied in section III. Section IV analyzes the performance of the estimators: their first order bias and variances are calculated in section IV-A, then their asymptotic expansions are derived in section IV-B (in the case of undamped single poles). These developments are illustrated in section V-A, where the variances of the estimators are compared to the Cramér-Rao bounds, and the generalized ESPRIT algorithm is applied to ARMA filter synthesis in section V-B. The main conclusions of this paper are summarized in section VI. Finally, the mathematical developments for the perturbation analysis are provided in the Appendix.

\section{GENERAL FRAMEWORK}

In sections II-A and II-B, we summarize the basics of the PACE model, also called quasipolynomial model, and the generalized ESPRIT algorithm, which were presented in [2].

\section{A. Polynomial Amplitude Complex Exponentials}

Definition 1. Let $K \in \mathbb{N}^{*}$. For all $k \in\{0 \ldots K-1\}$, define the partial order $M_{k} \in \mathbb{N}^{*}$, the frequency $\left.\left.f_{k} \in\right]-\frac{1}{2}, \frac{1}{2}\right]$, the damping (or amplifying) factor $\delta_{k} \in \mathbb{R}$, and the complex pole $z_{k}=e^{\delta_{k}+i 2 \pi f_{k}}$. Suppose that the complex poles are distinct from one another. Then a discrete signal $s(t)$ satisfies the PACE model of order $r \triangleq \sum_{k=0}^{K-1} M_{k}$ if and only if it can be written in the form

$$
s(t)=\sum_{k=0}^{K-1} \alpha_{k}[t] z_{k}^{t}
$$

where $\forall k \in\{0, \ldots, K-1\}, \alpha_{k}[t]$ is a complex polynomial of order $M_{k}-1$.

The polynomial $\alpha_{k}[t]$ can be decomposed onto the polynomial basis of falling factorials:

Definition 2 (Falling factorial). For all $m \in \mathbb{Z}$, the falling factorial of order $m$ is the polynomial ${ }^{1}$

$$
F_{m}[X]= \begin{cases}0 & \text { if } m<0 \\ 1 & \text { if } m=0 \\ \frac{1}{m !} \prod_{m^{\prime}=0}^{m-1}\left(X-m^{\prime}\right) & \text { if } m>0 .\end{cases}
$$

In this basis, equation (1) can be rewritten in the form

$$
s(t)=\sum_{k=0}^{K-1} \sum_{m=0}^{M_{k}-1} \alpha_{k}^{(m)} F_{m}[t] z_{k}^{t-m}
$$

where $\forall k \in\{0 \ldots K-1\}, \forall m \in\left\{0, M_{k}-1\right\}, \alpha_{k}^{(m)}$ is a complex amplitude. Define the real amplitude $a_{k}^{(m)}=\left|\alpha_{k}^{(m)}\right|$, and the phase ${ }^{2} \phi_{k}^{(m)}=\Im\left(\ln \left(\alpha_{k}^{(m)}\right)\right)$.

The PACE model can be characterized by means of matrix analysis. Indeed, the samples of the discrete signal $s(t)$ can be arranged into a Hankel data matrix with $n>r$ rows and $l \geq r$ columns:

$$
\boldsymbol{S}=\left[\begin{array}{cccc}
s(t-l+1) & \cdots & s(t-1) & s(t) \\
s(t-l+2) & \cdots & s(t) & s(t+1) \\
\vdots & \cdots & \vdots & \vdots \\
s(t-l+n) & \cdots & s(t+n-2) & s(t+n-1)
\end{array}\right]
$$

In particular, the range space of $S$ can be characterized by the generalized Pascal and Pascal-Vandermonde matrices.

Definition 3 (Generalized Pascal matrices). Let $z \in \mathbb{C}$ and $M \in \mathbb{N}^{*}$. The generalized Pascal matrix denoted $C_{M}^{n}(z)$ is an $n \times M$ matrix whose coefficients are $C_{M}^{n}(z)_{(i, j)}=F_{j}[i] z^{i-j}$ for all $i \in\{0 \ldots n-1\}$ and $j \in\{0 \ldots M-1\}$.

Example 4. If $M=3$ and $n=6$,

$$
C_{3}^{6}(z)=\left[\begin{array}{lll}
1 & 0 & 0 \\
z & 1 & 0 \\
z^{2} & 2 z & 1 \\
z^{3} & 3 z^{2} & 3 z \\
z^{4} & 4 z^{3} & 6 z^{2} \\
z^{5} & 5 z^{4} & 10 z^{3}
\end{array}\right]
$$

Definition 5 (Pascal-Vandermonde matrices). The $n \times r$ Pascal-Vandermonde matrix is the matrix formed by concatenating the generalized Pascal matrices $C_{k}^{n}=C_{M_{k}}^{n}\left(z_{k}\right)$ :

$$
\boldsymbol{V}^{n}=\left[\boldsymbol{C}_{0}^{n}, \ldots, \boldsymbol{C}_{(K-1)}^{n}\right] .
$$

Based on the above definitions, the following proposition, which is proved in [2], shows a factorization of the Hankel data matrix ${ }^{3}$ :

\footnotetext{
${ }^{1}$ Note that this definition does not match the classical definition of the falling factorial [25], [26], from which the multiplicative factor $\frac{1}{m !}$ is missing.

${ }^{2}$ In the whole paper, the notation $\ln ($.$) denotes the determination of the$ complex logarithm which corresponds to an angle lying in $]-\pi, \pi[$.

${ }^{3}$ In linear systems realization theory, state space representations also lead to low-rank factorizations of Hankel matrices [27].
} 
Proposition 1 (Factorization of the data matrix). An $n \times l$ Hankel matrix $\boldsymbol{S}$ of the form (3) where $s(t)$ is the signal defined in equation (2) can be factorized in the form

$$
\boldsymbol{S}=\boldsymbol{V}^{n} \boldsymbol{D} \boldsymbol{V}^{l^{T}}
$$

where $\boldsymbol{V}^{n}$ and $\boldsymbol{V}^{l}$ are the $n \times r$ and $l \times r$ Pascal-Vandermonde matrices, and $\boldsymbol{D}$ is an $r \times r$ block-diagonal matrix

$$
\boldsymbol{D}=\operatorname{diag}\left(\boldsymbol{H}_{0} \ldots \boldsymbol{H}_{(K-1)}\right)
$$

whose $k^{\text {th }}$ block $\boldsymbol{H}_{k}$ is an $M_{k} \times M_{k}$ upper anti-triangular Hankel matrix (in the particular case $M_{k}=1, \boldsymbol{H}_{k}=$ $\left.z_{k}^{t-l+1} \alpha_{k}^{(0)}\right)$.

\section{B. Estimation of the model parameters}

Proposition 1 shows that the matrix $S$ has rank $r$, and that its range space, called signal subspace, is also spanned by the Pascal-Vandermonde matrix $\boldsymbol{V}^{n}$.

1) Rotational Invariance Property: The ESPRIT method relies on a particular property of Vandermonde matrices known as rotational invariance [1], which reflects the invariance of the signal subspace to time shifts. Theorem 2, shown in [2], generalizes this property to Pascal-Vandermonde matrices.

Theorem 2 (Rotational Invariance Property of Pascal-Vandermonde matrices). Let $\boldsymbol{V}_{\downarrow}^{n}$ be the matrix extracted from $\boldsymbol{V}^{n}$ by deleting the last row. Similarly, let $\boldsymbol{V}_{\uparrow}^{n}$ be the matrix extracted from $\boldsymbol{V}^{n}$ by deleting the first row. Then $\boldsymbol{V}_{\downarrow}^{n}$ and $\boldsymbol{V}_{\uparrow}^{n}$ span the same subspace, and

$$
\boldsymbol{V}_{\uparrow}^{n}=\boldsymbol{V}_{\downarrow}^{n} \boldsymbol{J}
$$

where $\boldsymbol{J}$ is the $r \times r$ block-diagonal matrix

$$
\boldsymbol{J}=\operatorname{diag}\left(\boldsymbol{J}_{0}, \ldots, \boldsymbol{J}_{(K-1)}\right)
$$

whose $k^{\text {th }}$ block $\boldsymbol{J}_{k}$ is the $M_{k} \times M_{k}$ Jordan block whose diagonal coefficients are equal to $z_{k}$.

The interesting fact in theorem 2 is that equation (5) involves a Jordan matrix ${ }^{4} \boldsymbol{J}$, which characterizes the poles $z_{k}$ and their multiplicity $M_{k}$. As shown below, the generalized ESPRIT algorithm consists in computing $\boldsymbol{J}$ as a by-product of the Jordan canonical decomposition of a so-called spectral matrix.

2) The generalized ESPRIT method: In practice, the PascalVandermonde matrix $\boldsymbol{V}^{n}$ is unknown. Nevertheless, it was shown in [2] that in presence of white noise an $n \times r$ orthonormal matrix $\boldsymbol{W}$ spanning the signal subspace can be estimated by computing the left dominant $r$-dimensional singular subspace of the noisy data matrix, or by using subspace tracking methods [29]-[31]. Since $\boldsymbol{W}$ and $\boldsymbol{V}^{n}$ span the same subspace, there is an $r \times r$ invertible matrix $\boldsymbol{G}$ such that

$$
\boldsymbol{V}^{n}=\boldsymbol{W} \boldsymbol{G}
$$

Substituting equation (7) into equation (5) shows that $\boldsymbol{W}$ satisfies an equation similar to equation (5): $\boldsymbol{W}_{\uparrow}=\boldsymbol{W}_{\downarrow} \Phi$

\footnotetext{
${ }^{4}$ See [28, pp. 121-142] for a definition of Jordan canonical decomposition.
}

where $\Phi$, herein called the spectral matrix, is defined by its Jordan canonical decomposition:

$$
\Phi \triangleq G J G^{-1} .
$$

It can be noticed that $\forall t \in \mathbb{Z}$, the spectral matrix $\boldsymbol{\Phi}$, which depends on the observation window $\{t-l+1, \ldots, t+n-1\}$, is similar to the unique Jordan matrix $\boldsymbol{J}$. Finally, the generalized ESPRIT algorithm consists in:

- estimating a basis $\boldsymbol{W}$ of the signal subspace, via singular value decomposition for instance ${ }^{5}$,

- computing the spectral matrix ${ }^{6} \boldsymbol{\Phi}=\boldsymbol{W}_{\downarrow}^{\dagger} \boldsymbol{W}_{\uparrow}$.

- computing the eigenvalues of $\boldsymbol{\Phi}$ from which the estimated poles and their multiplicities can be extracted.

Note that in a noisy context, the estimated spectral matrix does not have multiple eigenvalues in practice, and the generalized ESPRIT algorithm cannot be applied as it is. This problem will be discussed in section A in the Appendix.

3) Estimation of the complex amplitudes: The complex amplitudes are estimated by means of the LS method. Let $\widehat{\boldsymbol{V}}^{N}$ be the $N \times r$ Pascal-Vandermonde matrix defined from the estimated poles, and $\widehat{s}$ the $N$-dimensional vector containing the successive samples of the observed signal. Then the LSestimate of the vector $\boldsymbol{\alpha}=\left[\boldsymbol{\alpha}_{0}, \ldots, \boldsymbol{\alpha}_{K-1}\right]^{T}$ (with $\boldsymbol{\alpha}_{k}=$ $\left.\left[\alpha_{k}^{(0)}, \ldots, \alpha_{k}^{\left(M_{k}-1\right)}\right]^{T}\right)$ containing the complex amplitudes is

$$
\widehat{\boldsymbol{\alpha}}=\widehat{\boldsymbol{V}}^{N \dagger} \widehat{\boldsymbol{s}}
$$

\section{Perturbation AnAlysis}

The objective here is to measure the performance of the estimators presented above in terms of bias and dispersion. Unfortunately, it is not possible to establish analytic formulae in the general case, because of the eigen or singular value decompositions. However, asymptotic results could be obtained by using the perturbation theory in the case of the sinusoidal model [23] and in the case of the ESM model [24], under the hypothesis of a high signal-to-noise ratio. We propose below to apply the perturbation theory in the more general framework of the PACE model, in order to finally derive the first and second moments of the estimators. First, we analyze the perturbation induced onto the frequencies and damping factors, from which we derive the perturbation induced onto the amplitudes and phases. The detailed mathematical developments can be found in the Appendix.

Suppose that the PACE signal $s(t)$ is corrupted by a perturbation $\varepsilon \Delta s(t)$ (where $\varepsilon<<1$ ), so that the observed signal is $s(t)+\varepsilon \Delta s(t)$. In other terms, the $n \times l$ Hankel data matrix $S$ is corrupted by an additive perturbation $\varepsilon \Delta S$, where $\Delta S$ is the $n \times l$ Hankel matrix containing the samples of $\Delta s(t)$, so that the observed matrix is

$$
\boldsymbol{S}(\varepsilon)=\boldsymbol{S}+\varepsilon \Delta \boldsymbol{S}
$$

${ }^{5}$ In linear systems realization theory, Ho's algorithm is a well-known method for identifying a state space representation [27]. The use of the singular value decomposition in this context was early proposed in [32].

${ }^{6}$ In the whole paper, the symbol $\dagger$ denotes the Moore-Penrose pseudoinverse. 
Then suppose that the generalized ESPRIT algorithm is applied to the perturbed matrix $S(\varepsilon)$ instead of the exact matrix $\boldsymbol{S}$. In section A in the Appendix, it is shown that the perturbed subspace weighting matrix $\boldsymbol{W}(\varepsilon)$ (section A1), spectral matrix $\boldsymbol{\Phi}(\varepsilon)$ (section A2), poles $z_{k}(\varepsilon)$, frequencies $f_{k}(\varepsilon)$ and damping factors $\delta_{k}(\varepsilon)$ (section A3) are $\mathcal{C}^{\infty}$ functions of $\varepsilon$ in the neighborhood of $\varepsilon=0$, leading to the first order expansions in proposition 3.

Proposition 3 (Perturbation of the frequencies and damping factors). Let ${ }^{7} \delta_{k}(\varepsilon)=\Re\left(\ln \left(z_{k}(\varepsilon)\right)\right)$ and $f_{k}(\varepsilon)=$ $\frac{1}{2 \pi} \Im\left(\ln \left(z_{k}(\varepsilon)\right)\right)$. Then the functions $\varepsilon \mapsto \delta_{k}(\varepsilon)$ and $\varepsilon \mapsto$ $f_{k}(\varepsilon)$ are $\mathcal{C}^{\infty}$ and admit the first order expansions:

$$
\left\{\begin{array}{l}
\delta_{k}(\varepsilon)=\delta_{k}+\varepsilon \Delta \delta_{k}+O\left(\varepsilon^{2}\right) \\
f_{k}(\varepsilon)=f_{k}+\varepsilon \Delta f_{k}+O\left(\varepsilon^{2}\right)
\end{array}\right.
$$

The first order perturbations $\Delta \delta_{k}$ and $\Delta f_{k}$ are of the form

$$
\left\{\begin{aligned}
\Delta \delta_{k} & =\frac{1}{M_{k}} \Re\left(\frac{\boldsymbol{u}_{k}{ }^{H} \Delta \boldsymbol{s}}{z_{k} \alpha_{k}^{\left(M_{k}-1\right)}}\right) \\
\Delta f_{k} & =\frac{1}{2 \pi M_{k}} \Im\left(\frac{\boldsymbol{u}_{k}^{H} \Delta \boldsymbol{s}}{z_{k} \alpha_{k}^{\left(M_{k}-1\right)}}\right),
\end{aligned}\right.
$$

where the vectors $\Delta s=[\Delta s(t-l+1), \ldots, \Delta s(t+n-1)]^{T}$ and $\boldsymbol{u}_{k}$ (whose coefficients, defined in equation (45) in the Appendix, depend on the model parameters) have dimension $N=n+l-1$.

Knowing the influence of a perturbation of the data onto the estimated poles, it is then possible to analyze the perturbation induced onto the amplitudes and phases, obtained from these poles by the least squares method. More precisely, it is shown in section B2 in the Appendix that the complex amplitudes $\alpha_{k}^{(m)}(\varepsilon)$, real amplitudes $a_{k}^{(m)}(\varepsilon)$ and phases $\phi_{k}^{(m)}(\varepsilon)$ are $\mathcal{C}^{\infty}$ functions of $\varepsilon$ in the neighborhood of $\varepsilon=0$, leading to the first order expansions in proposition 4.

Proposition 4 (Perturbation of the amplitudes and phases). Let $a_{k}^{(m)}(\varepsilon)=\left|\alpha_{k}^{(m)}(\varepsilon)\right|$ and $\phi_{k}^{(m)}(\varepsilon)=\frac{1}{2 \pi} \Im\left(\ln \left(\alpha_{k}^{(m)}(\varepsilon)\right)\right)$. If $a_{k}^{(m)} \neq 0$, then the functions $\varepsilon \mapsto a_{k}^{(m)}(\varepsilon)$ and $\varepsilon \mapsto \phi_{k}^{(m)}(\varepsilon)$ are $\mathcal{C}^{\infty}$ in the neighborhood of $\varepsilon=0$, and admit the first order expansion:

$$
\left\{\begin{array}{l}
a_{k}^{(m)}(\varepsilon)=a_{k}^{(m)}+\varepsilon \Delta a_{k}^{(m)}+O\left(\varepsilon^{2}\right) \\
\phi_{k}^{(m)}(\varepsilon)=\phi_{k}^{(m)}+\varepsilon \Delta \phi_{k}^{(m)}+O\left(\varepsilon^{2}\right) .
\end{array}\right.
$$

The first order perturbations $\Delta a_{k}^{(m)}$ and $\Delta \phi_{k}^{(m)}$ are of the form

$$
\left\{\begin{array}{l}
\Delta a_{k}^{(m)}=a_{k}^{(m)} \Re\left(\frac{\boldsymbol{b}_{k}^{(m) H} \Delta \boldsymbol{s}}{\alpha_{k}^{(m)}}\right) \\
\Delta \phi_{k}^{(m)}=\Im\left(\frac{\boldsymbol{b}_{k}^{(m)}{ }^{H} \Delta \boldsymbol{s}}{\alpha_{k}^{(m)}}\right),
\end{array}\right.
$$

where the vectors $\boldsymbol{b}_{k}^{(m)}$ (whose coefficients, defined in section B in the Appendix, depend on the model parameters) have dimension $N$.

\footnotetext{
${ }^{7}$ It is supposed here that all frequencies lie between $-\frac{1}{2}$ and $\frac{1}{2}$.
}

\section{Performance of the estimators}

Here we aim at exploiting the results of the perturbation analysis in section III to derive the first and second moments of the generalized ESPRIT estimators [2] in the case of a high signal-to-noise ratio. The most remarkable property shown below is that the asymptotic efficiency of the estimators of all parameters associated to single undamped poles is minimum if and only if the number of rows $n$ and the number of columns $l$ of the data matrix satisfy either $n=2 l$ or $l=2 n$. In practice, this result allows to properly dimension the data matrix when the length of the observation window $N=n+l-1$ is fixed.

From now on, we suppose that $\Delta s(t)$ is a circular complex stationary process of variance 1 . Then the stationary process $\varepsilon \Delta s(t)$ can be viewed as a complex noise of variance $\sigma^{2}=\varepsilon^{2}<<1$. Using vector notations, we can write $s(\varepsilon)=s+\varepsilon \Delta s$, where the vectors $s(\varepsilon), s$ and $\Delta s$ contain the $N$ successive samples of the corresponding signals in the observation window $\{t-l+1 \ldots t+n-1\}$. The covariance matrix $\Gamma \triangleq \mathbb{E}\left[\Delta s \Delta s^{H}\right]$ of the circular complex random vector $\Delta s$ is an $N \times N$ Toeplitz matrix, whose diagonal coefficients are equal to 1 . In the particular case of white noise, $\Gamma$ is equal to the identity.

Section IV-A presents the first order performance analysis, which is then simplified in section IV-B in an asymptotic context. The proofs of the results presented in this section can be found in the Appendix.

\section{A. First order performance}

The following proposition gives the variances of the estimated poles $\widehat{z}_{k}$, damping (or amplifying) factors $\widehat{\delta}_{k}$ and frequencies $\widehat{f}_{k}$, obtained by applying the generalized ESPRIT algorithm [2] to the perturbed signal $\widehat{s}=s(\varepsilon)$.

Proposition 5. The estimator $\widehat{z}_{k}=z_{k}(\varepsilon)$ of the pole $z_{k}$ is unbiased at the first order, and its variance is of the form

$$
\operatorname{var}\left(\widehat{z}_{k}\right) \sim \frac{\sigma^{2}}{\left(M_{k} a_{k}^{\left(M_{k}-1\right)}\right)^{2}} \boldsymbol{u}_{k}^{H} \boldsymbol{\Gamma} \boldsymbol{u}_{k} .
$$

where the vector $\boldsymbol{u}_{k}$ is defined in equation (45). In the same way, the estimators $\widehat{\delta}_{k}=\delta_{k}(\varepsilon)$ and $\widehat{f}_{k}=f_{k}(\varepsilon)$ of the damping factor $\delta_{k}$ and of the frequency $f_{k}$ are unbiased at the first order. Moreover, their respective variances are of the form

$$
\begin{aligned}
\operatorname{var}\left(\widehat{\delta}_{k}\right) & \sim \frac{\sigma^{2} e^{-2 \delta_{k}}}{2\left(M_{k} a_{k}^{\left(M_{k}-1\right)}\right)^{2}} \boldsymbol{u}_{k}{ }^{H} \boldsymbol{\Gamma} \boldsymbol{u}_{k} \\
\operatorname{var}\left(\widehat{f}_{k}\right) & \sim \frac{1}{4 \pi^{2}} \operatorname{var}\left(\widehat{\delta}_{k}\right) .
\end{aligned}
$$

The variances of $\widehat{\delta}_{k}$ and $\widehat{f}_{k}$ are derived from their first order expansions, presented in section A3 in the Appendix. A remarkable similitude can be noticed between their expressions and the Cramér-Rao bounds in equations (9) and (10). However equations (16) and (17) are not self-explanatory, because they involve many auxiliary variables, via the vectors $\boldsymbol{u}_{k}$. It will be shown in section IV-B that under some additional hypotheses, they can be simplified. 
These variances satisfy the following properties ${ }^{8}$ :

- if the noise is white $\left(\boldsymbol{\Gamma}=\boldsymbol{I}_{N}\right)$, the variances of $\widehat{\delta}_{k}$ and $\widehat{f}_{k}$ depend on the frequencies only by their differences ${ }^{9}$,

- if $z_{k}$ is a single pole, the variances of $\widehat{\delta}_{k}$ and $\widehat{f}_{k}$ do not depend on any phase, and they are inversely proportional to $a_{k}^{(0)^{2}}$, but they do not depend on any other amplitude ${ }^{10}$.

Proposition 6 below gives the variances of the estimated complex amplitudes $\widehat{\boldsymbol{\alpha}}$, real amplitudes $\widehat{a}_{k}^{(m)}$ and phases $\widehat{\phi}_{k}^{(m)}$, obtained by means of the Least Squares (LS) method [2].

Proposition 6. The estimator $\widehat{\boldsymbol{\alpha}}=\boldsymbol{\alpha}(\varepsilon)$ of the vector of complex amplitudes $\boldsymbol{\alpha}$ is unbiased at first order, and its covariance matrix is of the form

$$
\operatorname{cov}(\widehat{\boldsymbol{\alpha}}) \sim \sigma^{2} \boldsymbol{B}^{H} \boldsymbol{\Gamma} \boldsymbol{B},
$$

where the $N \times r$ matrix $\boldsymbol{B}$ is defined in equation (53). In the same way, the estimators $\widehat{a}_{k}^{(m)}=a_{k}^{(m)}(\varepsilon)$ and $\widehat{\phi}_{k}^{(m)}=\phi_{k}^{(m)}(\varepsilon)$ of the real-valued amplitude $a_{k}^{(m)}$ and the phase $\phi_{k}^{(m)}$ are unbiased at first order. Moreover, their respective variances are of the form

$$
\begin{aligned}
\operatorname{var}\left(\widehat{a}_{k}^{(m)}\right) & \sim \frac{\sigma^{2}}{2} \boldsymbol{b}_{k}^{(m)}{ }^{H} \boldsymbol{\Gamma} \boldsymbol{b}_{k}^{(m)} \\
\operatorname{var}\left(\widehat{\phi}_{k}^{(m)}\right) & \sim \frac{1}{a_{k}^{(m)^{2}}} \operatorname{var}\left(\widehat{a}_{k}^{(m)}\right),
\end{aligned}
$$

where the vectors $\boldsymbol{b}_{k}^{(m)}$, introduced in proposition 4 , are the columns of the matrix $\boldsymbol{B}$.

As for proposition 5, the variances of $\widehat{\alpha}_{k}, \widehat{a}_{k}$ and $\widehat{\phi}_{k}$ are obtained from their first order expansions, presented in section B2 in the Appendix.

\section{B. Asymptotic performance}

Let us suppose that the noise is white $\left(\boldsymbol{\Gamma}=\boldsymbol{I}_{N}\right)$ and that all poles are on the unit circle $\left(\forall k \in\{0 \ldots K-1\}, \delta_{k} \rightarrow 0\right)$. We present below some asymptotic expansions of the estimator variances with respect to $n, l$ and $N$.

Corollary 7. If $z_{k}$ is a single pole, the variances of the estimators $\widehat{\delta}_{k}$ and $\widehat{f}_{k}$ admit the expansions

$$
\begin{aligned}
& \operatorname{var}\left(\widehat{\delta}_{k}\right) \sim \frac{\sigma^{2}}{\max (n, l)^{2} \min (n, l) a_{k}^{(0)^{2}}}+O\left(\frac{1}{N^{4}}\right) \\
& \operatorname{var}\left(\widehat{f}_{k}\right) \sim \frac{\sigma^{2}}{4 \pi^{2} \max (n, l)^{2} \min (n, l) a_{k}^{(0)^{2}}}+O\left(\frac{1}{N^{4}}\right)
\end{aligned}
$$

Both of them are minimal for $n=2 l=\frac{2}{3}(N+1)$ or for $l=2 n=\frac{2}{3}(N+1)$ (if $N$ equals 2 modulo 3 ), and these minima admit the asymptotic expansions

$$
\begin{aligned}
\operatorname{var}\left(\widehat{\delta}_{k}\right) & \sim \frac{27}{4} \frac{\sigma^{2}}{N^{3} a_{k}^{(0)^{2}}} \\
\operatorname{var}\left(\widehat{f}_{k}\right) & \sim \frac{27}{4} \frac{\sigma^{2}}{4 \pi^{2} N^{3} a_{k}^{(0)^{2}}} .
\end{aligned}
$$

\footnotetext{
${ }^{8}$ These properties can be proved by a thorough analysis of the particular structure of the vector $\boldsymbol{u}_{k}$, defined in equation (45).

${ }^{9}$ This property is also satisfied by the Cramér-Rao bounds [12]

${ }^{10}$ In the case of the Cramér Rao bounds, this property also applies to multiple poles [12].
}

The proof of corollary 7 relies on the first order expansion of the vector $\boldsymbol{u}_{k}$ involved in equations (16) and (17), which admits a simple closed form. Attention must be paid to the fact that expressions (21) and (22) are only valid for a single pole. If $z_{k}$ is a multiple pole, these variances cannot be formulated in such a simple way and are function of the complex amplitudes associated to $z_{k}$ for all indices $m \in\left\{0 \ldots M_{k}-1\right\}$ (the optimal values of $n$ and $l$ are also function of these amplitudes in this case). These variances can be compared to the asymptotic Cramér-Rao bounds given in equations (13) and (15):

Under the above hypotheses, the asymptotic efficiency of the estimators of all damping factors and all frequencies associated to the single poles is the same one, independent from the model parameters, and equal to $9 / 8=1.125$ if $n=2 l$ or $l=2 n$.

In this way, the results obtained in [19] about the Matrix Pencil method (in the particular case of a single complex sinusoid) are recovered. Figure 1-a represents the ratio between the CramérRao bound and the variance of the estimators in a logarithmic scale, as a function of the ratio $\frac{n}{N+1}$. Thus it can be verified that the maximum is reached at $n=\frac{N+1}{3}$ and $n=\frac{2(N+1)}{3}$ as expected. Besides, the performance collapses when $n$ becomes too high or too small.
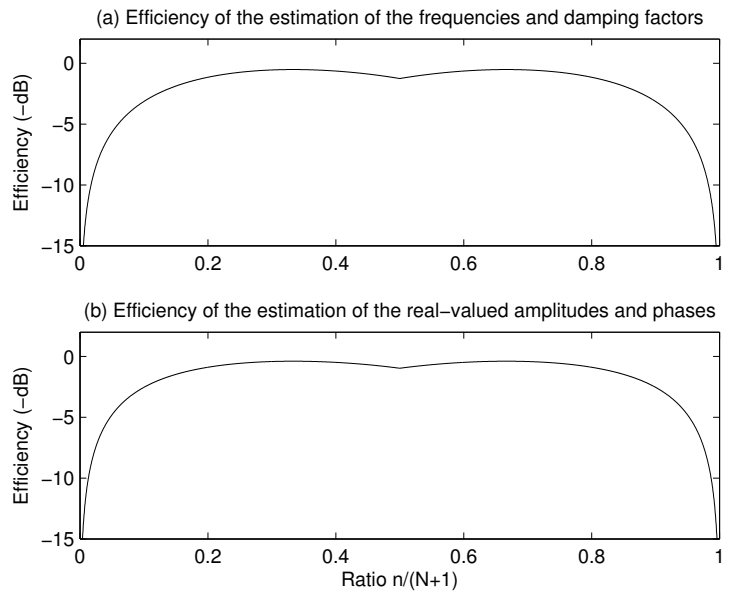

Fig. 1. Efficiency of the estimators

The following corollary is the analogue of corollary 7 for the real-valued amplitudes and phases.

Corollary 8. If $z_{k}$ is a single pole, the variances of the estimators $\widehat{a}_{k}^{(0)}$ and $\widehat{\phi}_{k}^{(0)}$ admit the expansions

$$
\begin{aligned}
\operatorname{var}\left(\widehat{a}_{k}^{(0)}\right) & \sim \frac{\sigma^{2}}{2}\left(\frac{1}{N}+\frac{N^{2}}{2 \max (n, l)^{2} \min (n, l)}\right)+O\left(\frac{1}{N^{2}}\right) \\
\operatorname{var}\left(\widehat{\phi}_{k}^{(0)}\right) & \sim \frac{\sigma^{2}}{2 a_{k}^{(0)}}\left(\frac{1}{N}+\frac{N^{2}}{2 \max (n, l)^{2} \min (n, l)}\right)+O\left(\frac{1}{N^{2}}\right) .
\end{aligned}
$$

Both of them are minimal for $n=2 l=\frac{2}{3}(N+1)$ or for $l=2 n=\frac{2}{3}(N+1)$ (if $N$ equals 2 modulo 3 ), and these 
minima admit the asymptotic expansions

$$
\begin{aligned}
\operatorname{var}\left(\widehat{a}_{k}^{(0)}\right) & \sim \frac{35}{16} \frac{\sigma^{2}}{N} \\
\operatorname{var}\left(\widehat{\phi}_{k}^{(0)}\right) & \sim \frac{35}{16} \frac{\sigma^{2}}{N a_{k}^{(0)^{2}}} .
\end{aligned}
$$

The proof of corollary 8 relies on the first order expansions of the vectors $\boldsymbol{b}_{k}^{(m)}$ involved in equations (19) and (20), which admit a simple closed form. Again, attention must be paid to the fact that expressions (23) and (24) are only valid for a single pole. These variances can be compared to the asymptotic Cramér-Rao bounds given in equations (16) and (17):

Under the above hypotheses, the asymptotic efficiency of the estimators of all the real-valued amplitudes and phases associated to single poles is the same one, independent from the model parameters, and equal to $35 / 32=1.09375$ if $n=2 l$ or $l=2 n$.

This efficiency is even better than that of the estimators $\widehat{\delta}_{k}$ and $\widehat{f}_{k}$. It can also be noticed that the optimum is obtained for the same values of $n$ and $l$ as in the previous case. Figure 1-b represents the ratio between the Cramér-Rao bound and the variance of the estimators in a logarithmic scale, as a function of the ratio $\frac{n}{N+1}$. Again, the maximum is reached at $n=\frac{N+1}{3}$ and $n=\frac{2(N+1)}{3}$, and the performance collapses when $n$ becomes too high or too small. The similitude between the curves represented in figures $1-\mathrm{a}$ and $1-\mathrm{b}$ is noticeable. This could be explained by the fact that the estimation of the amplitudes and the phases directly relies on the estimation of the frequencies and the damping factors.

\section{Simulation RESUlts}

A. Dependence of the variances with respect to the PACE parameters

This section illustrates the variations of the estimators variances with respect to the parameters of the PACE model. Note that propositions 5, 6 and corollaries 7, 8 show a rather simple dependency on the amplitudes and the variance $\sigma^{2}$. Therefore we focus here on the dependency on the frequency gap between two components (section V-A1), the damping factor (section V-A2), the spectral flatness of the noise (section V-A3), and the order of a pole (section V-A4). For these simulations, the same synthetic signals as those introduced in [12] are used. In the figures below, the solid lines represent the theoretical variance of the frequency estimators or that of the damping factor estimators, which are equivalent according to (17). In the same way, the dashed lines represent the theoretical relative variance of the amplitude estimators, which is equal to that of the phase estimators, according to (20).

1) Variation of the variances with respect to frequency gaps: We consider a signal of length $N=200$, composed of two undamped components $(K=2)$ of same order $M_{0}=M_{1}=1$, in white noise $\left(\boldsymbol{\Gamma}=\boldsymbol{I}_{N}\right.$ and $\left.\sigma^{2}=1\right)$. These components have zero phases, and same amplitudes, such that $\mathrm{SNR}_{0}^{(0)}=\mathrm{SNR}_{1}^{(0)}=50 \mathrm{~dB}$. Figures 2-a and 2-b show the variations of the variances of the estimators obtained with $n=\frac{2}{3}(N+1)$, with respect to the frequency gap $\left.\left.\Delta f=\left|f_{1}-f_{0}\right| \in\right] 0,0.5\right]$ ( $f_{0}$ was set to 0$)$. The diamonds represent the empirical variance, obtained by averaging 100 runs of the ESPRIT algorithm. They match the theoretical variance, which confirms the validity of our perturbation analysis for this SNR. The variation rate of the variances is similar to that of the Cramér Rao bounds [12]: it is broken at $\Delta f=\frac{1}{N}=510^{-3}$, which corresponds to the resolution limit of Fourier analysis. At this limit point, the relative variance of the amplitude estimate is still lower than $-60 \mathrm{~dB}$, which shows the good resolution of the ESPRIT algorithm. The efficiencies of both estimators are represented in figure 2-c. It can be noticed that they remain close to 1 , even when the frequency gap tends to zero.
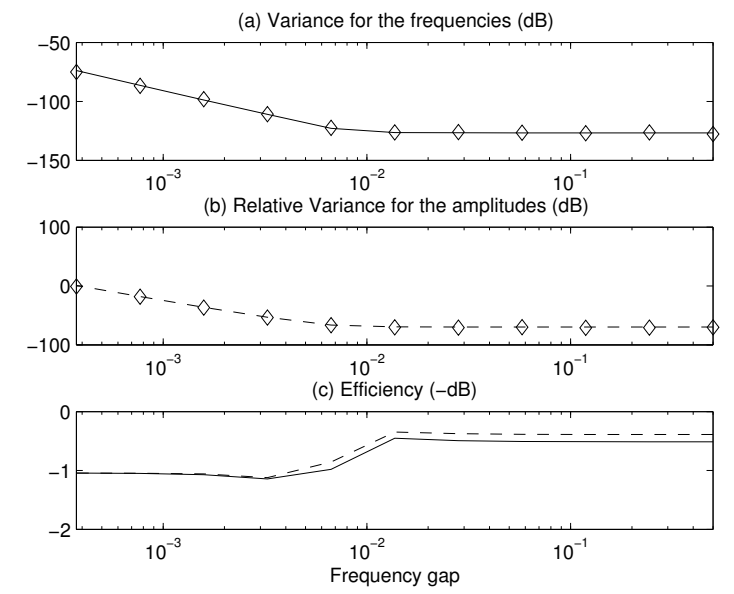

Fig. 2. Variation of the variances with respect to the frequency gap

2) Variation of the variances with respect to the damping factor: We consider a signal of length $N=100$, composed of one component $(K=1)$ of order $M_{0}=1$, in white noise $\left(\boldsymbol{\Gamma}=\boldsymbol{I}_{N}\right.$ and $\left.\sigma^{2}=1\right)$. This component has zero frequency and phase, and an amplitude such that $\mathrm{SNR}_{0}^{(0)}=50 \mathrm{~dB}$. Figures 3-a and 3-b show the variations of the variances of the estimators obtained with $n=\frac{2}{3}(N+1)$, with respect to the damping factor $\delta_{0}$. The diamonds represent the empirical variance, obtained by averaging 500 runs of the ESPRIT algorithm. They no longer exactly match the theoretical variance for negative values of delta, which shows the validity limit of our perturbation analysis with respect to the high SNR hypothesis. Again, these variations are very similar to those of the Cramér-Rao bounds illustrated in [12]. The efficiencies of both estimators are represented in figure 3-c. They remain close to 1 whatever the value of the damping factor is.

3) Variation of the variances with respect to the spectral flatness of the noise: We consider a signal of length $N=100$, composed of one undamped component $(K=1)$ of order $M_{0}=1$, in colored noise. This component has a zero phase, a normalized frequency equal to 0.05 , and an amplitude such that $\mathrm{SNR}_{0}^{(0)}=50 \mathrm{~dB}$. The noise is obtained by filtering a 

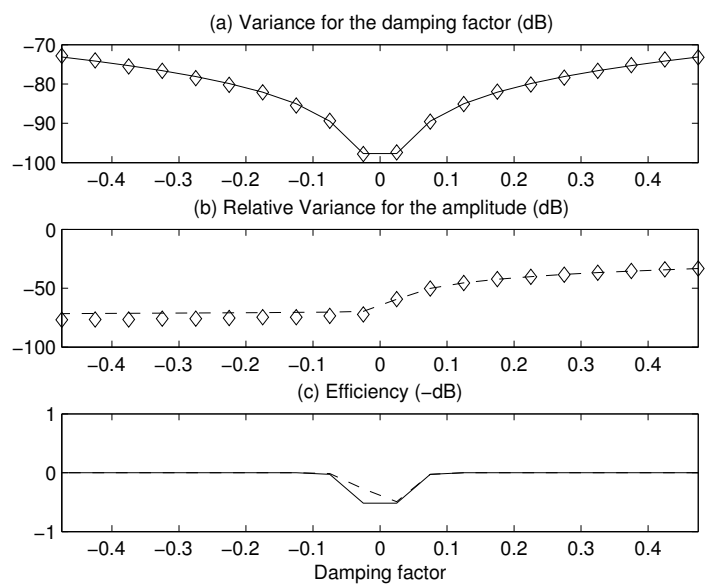

Fig. 3. Variation of the variances with respect to the damping factor

white noise by the filter of transfer function $H_{a}(z)=\frac{1}{1-a z^{-1}}$ (where $0 \leq a<1)$, such that $\Gamma=\operatorname{Toeplitz}\left(1, a, a^{2} \ldots a^{N-1}\right)$. The Spectral Flatness (SF) measure of the noise is defined as

$$
\mathrm{SF}(a)=\frac{\exp \left(\int_{0}^{1} \ln \left(\left|H_{a}\left(e^{i 2 \pi f}\right)\right|^{2}\right) \mathrm{d} f\right)}{\int_{0}^{1}\left|H_{a}\left(e^{i 2 \pi f}\right)\right|^{2} \mathrm{~d} f} .
$$

By tuning the parameter $a$, it is possible to make the spectral flatness map the range 10,1$]$ (the case $\mathrm{SF}=1$ corresponds to white noise). Figure 4 illustrates the variations of the variances of the estimators obtained with $n=\frac{2}{3}(N+1)$, with respect to the spectral flatness of the noise. As expected, figure 4-c shows that the efficiency degrades when the spectral flatness decreases (note that the ESPRIT algorithm explicitly relies on the white noise assumption). In other respects, figures 4-a and 4-b show that the variances admit a maximum when $\mathrm{SF} \simeq 0.5$. At this point, the theoretical and the empirical variances (obtained by averaging 1000 runs) no longer match in figure 4-b, which shows the validity limit of our perturbation analysis for this SNR. In the range $\mathrm{SF} \in[0.5,1]$, the variances decrease when the spectral flatness increases, as expected. In the range $\mathrm{SF} \in] 0.02,0.5]$, we observe the inverse phenomenon. Indeed, as mentioned in [12], the power spectral density of the noise becomes a sharp peak when SF becomes low, and converges to a spectral line when $\mathrm{SF} \rightarrow 0$. Therefore the problem of estimating the single undamped component in colored noise becomes close to the problem of estimating two undamped components without noise. However, contrary to what is observed in [12] in the case of the Cramér-Rao bounds, we note that the variances are stationary in the interval SF $\in] 0,0.02]$. This is because the ESPRIT algorithm is applied with an "erroneous" model order $(K=1 \text { instead of } 2)^{11}$.

4) Variation of the variances with respect to the pole order: We consider a signal of length $N=20$, composed of one undamped component $(K=1)$ of order $M_{0} \in\{1 \ldots 4\}$, in white noise $\left(\boldsymbol{\Gamma}=\boldsymbol{I}_{N}\right.$ and $\left.\sigma^{2}=1\right)$. This component has

\footnotetext{
${ }^{11}$ The impact of an erroneous modeling order on the estimated parameters was studied in [33].
}
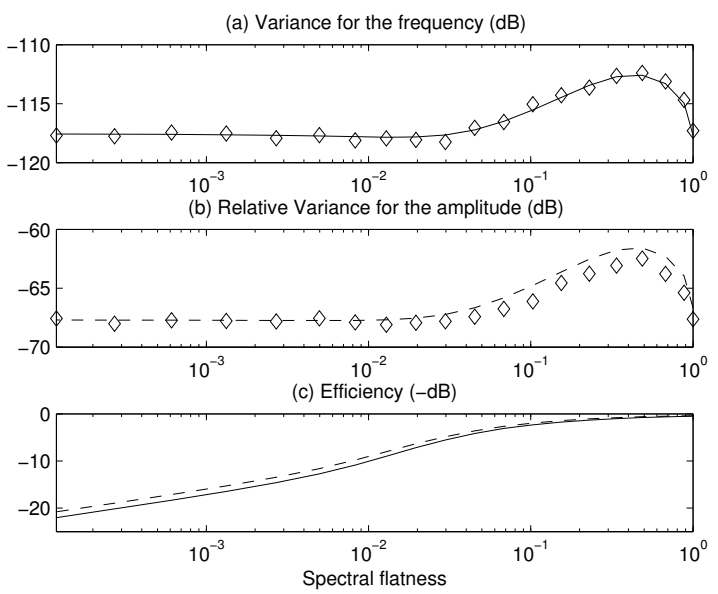

Fig. 4. Variation of the variances with respect to the spectral flatness of the noise

zero phases, and amplitudes such that $\mathrm{SNR}_{0}^{\left(M_{0}-1\right)}=50 \mathrm{~dB}$, and $\forall m<M_{0}-1, \mathrm{SNR}_{0}^{(m)}=0$. The corresponding pole is $z_{0}=1$. Figures $5-\mathrm{a}$ and $5-\mathrm{b}$ show the variations of the variances of the estimators obtained with $n=\frac{2}{3}(N+1)$, with respect to the pole order $M_{0}$. They confirm the results obtained for the Cramér-Rao bounds [12]: estimating multiple poles is all the more difficult as their order is high. Actually this estimation is no longer possible in this case if $M_{0}>4$. Besides, the empirical variance (obtained by averaging 100 runs) and the theoretical variance no longer exactly match for $M_{0} \geq 4$, which shows the validity limit of our perturbation analysis for this SNR (we observed that they match again if the SNR becomes greater than $80 \mathrm{~dB}$ ). In other respects, figure 5-c shows that the efficiency rapidly degrades when $M_{0}$ increases. We may infer that the arithmetic mean of the scattered eigenvalues is not a sufficiently reliable estimator for a pole of high multiplicity. Some ideas to improve this estimator are suggested in [34].
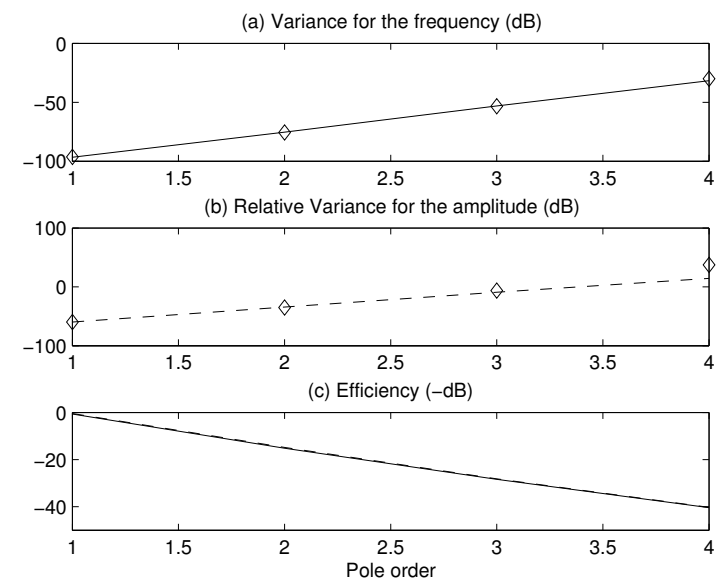

Fig. 5. Variation of the variances with respect to the pole order 


\section{B. Application to ARMA filter synthesis}

As shown in section $\mathrm{V}$-A4, estimating multiple poles is a difficult task. In order to illustrate the practical capabilities of the generalized ESPRIT algorithm in presence of multiple poles, we propose below an application to ARMA filter synthesis, in the context of system conversion from continuous time to discrete time.

1) Principle: We consider continuous time systems defined by an ordinary differential equation (ODE) with real-valued coefficients (below $\tau$ denotes the continuous time, in seconds) $a_{0} \widetilde{y}(\tau)+a_{1} \widetilde{y}^{\prime}(\tau)+\ldots+a_{p} \widetilde{y}^{(p)}(\tau)=b_{0} \widetilde{x}(\tau)+\ldots+b_{q} \widetilde{x}^{(q)}(\tau)$, whose transfer function can be written in the form

$$
\widetilde{H}(\rho)=\frac{\sum_{k=0}^{q} b_{k} \rho^{k}}{\sum_{k=0}^{p} a_{k} \rho^{k}}
$$

where $\rho=i 2 \pi \nu$ and $\nu$ denotes the frequency, in Hertz. Moreover, we suppose that $\widetilde{x}(\tau)$ and $\widetilde{y}(\tau)$ satisfy the conditions of the Nyquist-Shannon sampling theorem: $\exists T>0$ such that the Fourier transforms $\widetilde{X}(\nu)$ and $\widetilde{Y}(\nu)$ are zero outside the range $\left[-\frac{1}{2 T}, \frac{1}{2 T}\right]$, and we consider the discrete signals $x(t)=\widetilde{x}(t T)$ and $y(t)=\widetilde{y}\left(\left(t-t_{0}\right) T\right.$ ) for all $t \in \mathbb{Z}$ (where $t_{0} \in \mathbb{R}$ ), obtained by sampling $x(\tau)$ and $y(\tau)$ at the frequency $1 / T$. Then it is well known that $y(t)$ can be obtained from $x(t)$ by applying the discrete filter of frequency response

$$
H\left(e^{i 2 \pi f}\right)=\widetilde{H}\left(i 2 \pi \frac{f}{T}\right) e^{-i 2 \pi t_{0} f}=\frac{\sum_{k=0}^{q} b_{k}\left(i 2 \pi \frac{f}{T}\right)^{k}}{\sum_{k=0}^{p} a_{k}\left(i 2 \pi \frac{f}{T}\right)^{k}} e^{-i 2 \pi t_{0} f}
$$

where $f \in\left[-\frac{1}{2}, \frac{1}{2}\right]$ is the normalized frequency. It can be noticed that the time delay $t_{0}$ can be chosen such that $H\left(e^{i 2 \pi \frac{1}{2}}\right)=H\left(e^{-i 2 \pi \frac{1}{2}}\right)$. In this case, if the denominator is never zero, the 1-periodic function $f \mapsto H\left(e^{i 2 \pi f}\right)$ is continuous and piecewise continuously differentiable, which proves that $h(t)=O\left(\frac{1}{t^{2}}\right)$, thus the discrete filter $h$ is stable. The impulse response $h(t)$ can then be obtained by numerically computing the inverse discrete time Fourier transform of $H\left(e^{i 2 \pi f}\right)$. However this impulse response $h(t)$ is generally infinite, and we aim at approximating it by an AutoRegressive Moving Average (ARMA) filter $g(t)$.

It is well known that the general rational transfer function of a stable ARMA filter can be decomposed in the form

$$
\begin{aligned}
G(z)= & \sum_{m=0}^{M_{0}-1} \alpha_{0}^{(m)} z^{-m}+\sum_{0<\left|z_{k}\right|<1} \sum_{m=0}^{M_{k}-1} \frac{\alpha_{k}^{(m)}}{\left(1-z_{k} z^{-1}\right)^{(1+m)}} \\
& +\sum_{1<\left|z_{k}\right|<+\infty} \sum_{m=0}^{M_{k}-1} \frac{\beta_{k}^{(m)}\left(-z / z_{k}\right)^{(1+m)}}{\left(1-z / z_{k}\right)^{(1+m)}}
\end{aligned}
$$

where for all $k \in\{0 \ldots K-1\} z_{k}$ is a pole of multiplicity $M_{k}$ (here we assume $z_{0}=0$ ). This transfer function corresponds to the impulse response

$$
g(t)=\sum_{0 \leq\left|z_{k}\right|<1} \sum_{m=0}^{M_{k}-1} \alpha_{k}^{(m)} F_{m}(t) z_{k}^{t-m}
$$

for all $t \geq 0$, and

$$
g(t)=\sum_{1<\left|z_{k}\right|<+\infty} \sum_{m=0}^{M_{k}-1}(-1)^{(1+m)} \beta_{k}^{(m)} F_{m}(|t|-1) z_{k}^{-|t|}
$$

for all $t<0$.

Thus both the causal and anticausal parts of $g(t)$ satisfy a PACE model ${ }^{12}$. Following this remark, we can find an ARMA filter $g$ which approximates the discrete filter $h$ by applying the generalized ESPRIT algorithm to the impulse response $h(t)$ on two appropriately chosen finite intervals.

2) Example: The ARMA filter synthesis method could be successfully used for designing differentiator or integrator filters. Here it is applied to the continuous time system

$$
\widetilde{y}(\tau)+3 \widetilde{y}^{\prime}(\tau)+3 \widetilde{y}^{\prime \prime}(\tau)+\widetilde{y}^{\prime \prime \prime}(\tau)=\widetilde{x}(\tau)-\widetilde{x}^{\prime}(\tau),
$$

with parameters $T=1$ and $^{13} t_{0} \simeq 2.3924$. The impulse and the frequency response of the corresponding discrete filter $h$ are represented in figure 6 .
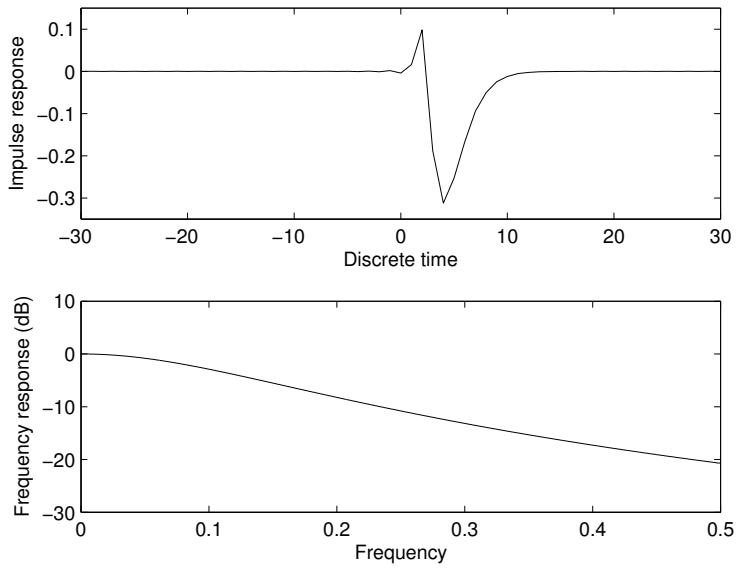

Fig. 6. Impulse and frequency response of the discrete filter

Equation (26) shows that the continuous time filter contains a triple pole at $\rho=-1$. When synthesizing the corresponding ARMA filter, we thus expect to find a triple pole at $z=$ $e^{\frac{\rho}{T}}=1 / e$. Figure 7 represents the estimated poles of the causal part of $g$ in the complex plane, obtained by applying the ESPRIT algorithm with $N=128, n=(N+1) / 3=$ 43 , and ${ }^{14} r=15$. As expected, we observe a triple pole in the neighborhood of $1 / e \simeq 0.3679$ (which is scattered into three single eigenvalues forming the vertices of an equilateral triangle). We also observe a double pole in the neighborhood of 0 , which corresponds to the polynomial part of the transfer function in equation (25). Finally, we obtained an ARMA filter $g$ with 26 poles and 27 zeros. The approximation error for

\footnotetext{
${ }^{12}$ Note that the causal part generally contains a multiple pole $z_{0}=0$, whereas the anticausal part never contains a pole at $z=\infty$.

${ }^{13}$ The fractional part of the delay $t_{0}$ was chosen in order to make the frequency response $H\left(e^{i 2 \pi f}\right)$ continuous, and the integer part of $t_{0}$ was chosen as the smallest integer such that the anticausal part of the estimated filter $g$ has no pole at $z=\infty$.

${ }^{14}$ The order $r$ was selected by means of Information Theoretic Criteria [35].
} 
the impulse response was $\max _{n \in[-128,128]}|h(n)-g(n)|<510^{-8}$, and the approximation error for the frequency response was $\max _{f \in\left[-\frac{1}{2}, \frac{1}{2}\right]}\left|20 \log _{10} H\left(e^{i 2 \pi f}\right)-20 \log _{10} G\left(e^{i 2 \pi f}\right)\right|<10^{-5}$.

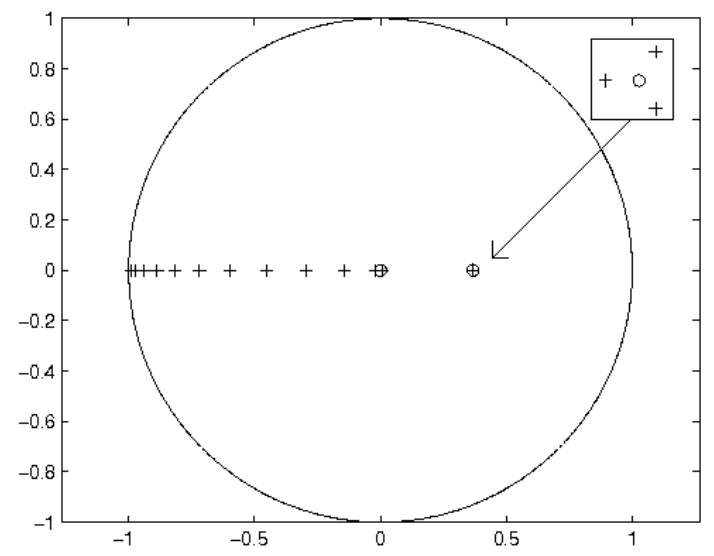

Fig. 7. Poles of the causal part of the ARMA filter

\section{CONCLUSIONS}

In this paper, the performance of the generalized ESPRIT algorithm for estimating the parameters of the PACE model, also called quasipolynomial model, was investigated in the context of high SNRs. This work was based on the analysis of the first order perturbations induced by an additive noise. In particular, it was shown that the perturbation of the estimated poles is not sensitive to the particular choice of the orthonormal subspace weighting matrix. In other respects, the presence of noise scatters multiple poles into several single eigenvalues, forming the vertices of a regular polygon. However it was proved that the estimation of multiple poles can be improved by calculating the arithmetic mean of the scattered eigenvalues.

Then it was shown that the estimators of all the parameters obtained in this way are unbiased, and their variances were calculated and compared to the Cramér-Rao bounds. By supposing that the noise is white, that all poles are on the unit circle, and that the SNR, $n$ and $l \rightarrow+\infty$, it was shown that the efficiency of single poles estimators is close to 1 . More precisely, the asymptotic efficiency of the estimators of all damping factors and frequencies is the same one, independent from the model parameters, and equal to $9 / 8=1.125$ if $n=2 l$ or $l=2 n$. In other respects, the asymptotic efficiency of the estimators of all the real-valued amplitudes and phases is the same one, independent from the model parameters, and equal to $35 / 32=1.09375$ if $n=2 l$ or $l=2 n$.

However, our simulation results showed that the variances of the estimators associated to a multiple pole present an exponential increase with the order of the pole. Thus the practical estimation of the PACE model parameters is only possible if the exponentials are modulated by polynomials of low order. Nevertheless, some recent advances in linear algebra computations, such as the techniques proposed in [34], offer interesting outlooks for improving the estimation of multiple poles.

\section{APPENDIX}

The following developments lead to propositions 3 and 4 presented in section III. The exhaustive proofs of these results are presented in a supporting document which is available at http://www.tsi.enst.fr/〜rbadeau/.

\section{A. Perturbation of the frequencies and damping factors}

Below, we successively derive the following first order expansions:

- $\boldsymbol{W}(\varepsilon)=\boldsymbol{W}+\varepsilon \Delta \boldsymbol{W}+O\left(\varepsilon^{2}\right)$, where the expression of $\Delta \boldsymbol{W}$ is a linear function of $\Delta \boldsymbol{S}$ (proposition 9);

- $\boldsymbol{\Phi}(\varepsilon)=\boldsymbol{\Phi}+\varepsilon \Delta \boldsymbol{\Phi}+O\left(\varepsilon^{2}\right)$, where the expression of $\Delta \boldsymbol{\Phi}$ is a linear function of $\Delta \boldsymbol{W}$ (proposition 10);

- $z_{k}(\varepsilon)=z_{k}+\varepsilon \Delta z_{k}+O\left(\varepsilon^{2}\right)$, where the expression of $\Delta z_{k}$ is a linear function of $\Delta \Phi$ (proposition 12);

- $\left\{\begin{array}{l}\delta_{k}(\varepsilon)=\delta_{k}+\varepsilon \Delta \delta_{k}+O\left(\varepsilon^{2}\right) \\ f_{k}(\varepsilon)=f_{k}+\varepsilon \Delta f_{k}+O\left(\varepsilon^{2}\right)\end{array}\right.$ where the expressions of $\Delta \delta_{k}$ and $\Delta f_{k}$ are functions of $\Delta z_{k}$ (corollary 3 ).

Finally, successive substitutions lead to the expressions of $\Delta \delta_{k}$ and $\Delta f_{k}$ as functions of the additive perturbation $\Delta s$.

1) Perturbation of the signal subspace: Here we analyze the influence of a perturbation of the data onto the signal subspace. For all $\varepsilon$, let $\Pi(\varepsilon)$ be the $n \times n$ projector onto the $r$-dimensional dominant subspace of the $n \times n$ positive semidefinite matrix $\boldsymbol{S}(\varepsilon) \boldsymbol{S}(\varepsilon)^{H}$. If $\boldsymbol{W}$ is orthonormal, $\Pi(0)=\boldsymbol{W} \boldsymbol{W}^{H}$. Then the perturbation theory shows that the function $\varepsilon \mapsto \Pi(\varepsilon)$ is $\mathcal{C}^{\infty}$ in a neighborhood of $\varepsilon=0$. In the literature, the asymptotic performance analysis of some subspacebased algorithms was performed by investigating the perturbation of this projector at the first order [36]. However we are interested here in the perturbation of the subspace weighting matrix $W$, which is analyzed in the following proposition.

Proposition 9 (Perturbation of the signal subspace). There exists an infinity ${ }^{15}$ of $\mathcal{C}^{\infty}$ functions $\varepsilon \mapsto \boldsymbol{W}(\varepsilon)$ defined in a neighborhood of $\varepsilon=0$ and with values in the group of complex orthonormal matrices $\mathbb{U}^{n \times r}$, which span the r-dimensional dominant subspace of $\boldsymbol{S}(\varepsilon)$ (i.e. such that $\boldsymbol{W}(0)=\boldsymbol{W}$ and $\boldsymbol{W}(\varepsilon) \boldsymbol{W}(\varepsilon)^{H}=\boldsymbol{\Pi}(\varepsilon)$ ). Each $\mathcal{C}^{\infty}$ function $\varepsilon \mapsto W(\varepsilon)$ admits a first order expansion

$$
\boldsymbol{W}(\varepsilon)=\boldsymbol{W}+\varepsilon \Delta \boldsymbol{W}+O\left(\varepsilon^{2}\right) .
$$

The first order perturbation $\Delta \boldsymbol{W}$ can be decomposed as

$$
\Delta \boldsymbol{W}=\Delta \boldsymbol{W}^{\perp}-\boldsymbol{W} \boldsymbol{A},
$$

where the $r \times r$ matrix $\boldsymbol{A} \triangleq \Delta \boldsymbol{W}^{H} \boldsymbol{W}$ is antihermitian and the $n \times r$ matrix $\Delta \boldsymbol{W}^{\perp}$ is orthogonal to $\operatorname{span}(\boldsymbol{W})$ :

$$
\Delta \boldsymbol{W}^{\perp}=\left(\boldsymbol{I}_{n}-\boldsymbol{W} \boldsymbol{W}^{H}\right) \Delta \boldsymbol{S} \boldsymbol{S}^{\dagger} \boldsymbol{W}
$$

The proof of proposition 9 relies on the implicit definition of $\boldsymbol{W}(\varepsilon)$ as the unique minimum point of a cost function $\mathcal{J}$. Equations (27) to (29) are thus derived by zeroing the first derivative of $\mathcal{J}$.

Proof of proposition 9:

It can be verified that the function

$$
\boldsymbol{W}(\varepsilon) \triangleq \Pi(\varepsilon) \boldsymbol{W}\left(\boldsymbol{W}^{H} \boldsymbol{\Pi}(\varepsilon) \boldsymbol{W}\right)^{-\frac{1}{2}}
$$

satisfies all the properties mentioned in proposition 9. It is also clear that any function of the form $\boldsymbol{W}(\varepsilon) \boldsymbol{\Theta}(\varepsilon)$, where $\varepsilon \mapsto \boldsymbol{\Theta}(\varepsilon)$ is a $\mathcal{C}^{\infty}$ function, whose values belong to the group of orthonormal matrices

${ }^{15}$ All these functions are obtained by right-multiplying any of them by a $\mathcal{C}^{\infty}$ function, with values in the group of complex orthonormal matrices $\mathbb{U}^{r \times r}$, and reaching the value $\boldsymbol{I}_{r}$ at $\varepsilon=0$. 
$\mathcal{O}_{r}(\mathbb{C})$, and which reaches the value $\boldsymbol{I}_{r}$ at $\varepsilon=0$, also satisfies these properties. Lastly, if $\varepsilon \mapsto W^{\prime}(\varepsilon)$ is an other function satisfying all these properties, then $\Pi(\varepsilon)=\boldsymbol{W}(\varepsilon) \boldsymbol{W}(\varepsilon)^{H}=\boldsymbol{W}^{\prime}(\varepsilon) \boldsymbol{W}^{\prime}(\varepsilon)^{H}$. Therefore $\boldsymbol{W}^{\prime}(\varepsilon)=\boldsymbol{W}(\varepsilon) \boldsymbol{\Theta}(\varepsilon)$, where $\boldsymbol{\Theta}(\varepsilon) \triangleq \boldsymbol{W}(\varepsilon)^{H} \boldsymbol{W}^{\prime}(\varepsilon)$ is a $\mathcal{C}^{\infty}$ function, whose values belong to the group of orthonormal matrices $\mathcal{O}_{r}(\mathbb{C})$ since $\boldsymbol{W}(\varepsilon)$ and $\boldsymbol{W}^{\prime}(\varepsilon)$ are two orthonormal bases of the same subspace, and which reach the value $\boldsymbol{W}^{H} \boldsymbol{W}=\boldsymbol{I}_{r}$ at $\varepsilon=0$. Then note that according to [37], any orthonormal matrix $\boldsymbol{W}(\varepsilon)$ spanning the principal subspace of the matrix $\boldsymbol{S}(\varepsilon) \boldsymbol{S}(\varepsilon)^{H}$ minimizes the function

$$
\begin{aligned}
\mathcal{J}: \mathbb{C}^{n \times r} & \rightarrow \mathbb{R} \\
\boldsymbol{U} & \mapsto \frac{1}{2}\left\|\boldsymbol{S}(\varepsilon)-\boldsymbol{U} \boldsymbol{U}^{H} \boldsymbol{S}(\varepsilon)\right\|_{F}^{2}
\end{aligned}
$$

Consequently, the gradient $\boldsymbol{\nabla} \mathcal{J}(\boldsymbol{U})$ is zero at $\boldsymbol{U}=\boldsymbol{W}(\varepsilon)$. However it can be verified ${ }^{16}$ that $\nabla \mathcal{J}(\boldsymbol{U})=$

$$
\left(-2 \boldsymbol{S}(\varepsilon) \boldsymbol{S}(\varepsilon)^{H}+\boldsymbol{S}(\varepsilon) \boldsymbol{S}(\varepsilon)^{H} \boldsymbol{U} \boldsymbol{U}^{H}+\boldsymbol{U} \boldsymbol{U}^{H} \boldsymbol{S}(\varepsilon) \boldsymbol{S}(\varepsilon)^{H}\right) \boldsymbol{U} .
$$

Let $\boldsymbol{W}(\varepsilon)=\boldsymbol{W}+\varepsilon \Delta \boldsymbol{W}+O\left(\varepsilon^{2}\right)$ be the first order expansion of the function $\varepsilon \mapsto \boldsymbol{W}(\varepsilon)$. Then

$$
\boldsymbol{\nabla} \mathcal{J}(\boldsymbol{W}(\varepsilon))=\varepsilon \boldsymbol{\nabla} \mathcal{J}^{(1)}+O\left(\varepsilon^{2}\right)
$$

where

$$
\begin{aligned}
\boldsymbol{\nabla} \mathcal{J}^{(1)}= & -\left(\boldsymbol{I}_{n}-\boldsymbol{W} \boldsymbol{W}^{H}\right) \Delta \boldsymbol{S} \boldsymbol{S}^{H} \boldsymbol{W} \\
& +\Delta \boldsymbol{W}\left(\boldsymbol{W}^{H} \boldsymbol{S} \boldsymbol{S}^{H} \boldsymbol{W}\right)+\boldsymbol{W} \boldsymbol{N}
\end{aligned}
$$

and

$$
\begin{aligned}
\boldsymbol{N} \triangleq & \left(\boldsymbol{W}^{H} \boldsymbol{S} \boldsymbol{S}^{H} \boldsymbol{W}\right)\left(\boldsymbol{W}^{H} \Delta \boldsymbol{W}+\Delta \boldsymbol{W}^{H} \boldsymbol{W}\right) \\
& +\Delta \boldsymbol{W}^{H} \boldsymbol{W}\left(\boldsymbol{W}^{H} \boldsymbol{S} \boldsymbol{S}^{H} \boldsymbol{W}\right) .
\end{aligned}
$$

However, the first order expansion of the orthonormality condition $\boldsymbol{W}(\varepsilon)^{H} \boldsymbol{W}(\varepsilon)=\boldsymbol{I}_{r}$ shows that $\boldsymbol{W}^{H} \Delta \boldsymbol{W}+\Delta \boldsymbol{W}^{H} \boldsymbol{W}=\mathbf{0}_{(r \times r)}$, which means that the matrix $\boldsymbol{A} \triangleq \Delta \boldsymbol{W}^{H} \boldsymbol{W}$ is antihermitian. Thus $\boldsymbol{N}=\boldsymbol{A}\left(\boldsymbol{W}^{H} \boldsymbol{S} \boldsymbol{S}^{H} \boldsymbol{W}\right)$. Since $\boldsymbol{\nabla} \mathcal{J}(\boldsymbol{W}(\varepsilon))=\mathbf{0}_{(n \times r)}$, equations (30) to (32) yield

$$
\Delta \boldsymbol{W}=\left(\boldsymbol{I}_{n}-\boldsymbol{W} \boldsymbol{W}^{H}\right) \Delta \boldsymbol{S} \boldsymbol{S}^{H} \boldsymbol{W}\left(\boldsymbol{W}^{H} \boldsymbol{S} \boldsymbol{S}^{H} \boldsymbol{W}\right)^{-1}-\boldsymbol{W} \boldsymbol{A} .
$$

Finally, by noting that $\boldsymbol{S}^{H} \boldsymbol{W}\left(\boldsymbol{W}^{H} \boldsymbol{S} \boldsymbol{S}^{H} \boldsymbol{W}\right)^{-1}=\boldsymbol{S}^{\dagger} \boldsymbol{W}$, equations (27) to (29) can be derived.

2) Perturbation of the spectral matrix: The following proposition complements the result of proposition 9 by showing how the spectral matrix is perturbed.

Proposition 10 (Perturbation of the spectral matrix). Suppose that the matrix $\boldsymbol{W}_{\downarrow}$ is full-rank. Then in the neighborhood of $\varepsilon=0$, $W_{\downarrow}(\varepsilon)$ is also full-rank. Moreover, the function

$$
\boldsymbol{\Phi}(\varepsilon) \triangleq \boldsymbol{W}(\varepsilon)_{\downarrow}^{\dagger} \boldsymbol{W}(\varepsilon)_{\uparrow}
$$

is $\mathcal{C}^{\infty}$, and admits the first order expansion:

$$
\boldsymbol{\Phi}(\varepsilon)=\mathbf{\Phi}+\varepsilon \Delta \boldsymbol{\Phi}+O\left(\varepsilon^{2}\right) .
$$

The first order perturbation $\Delta \mathbf{\Phi}$ can be written in the form

$$
\Delta \boldsymbol{\Phi}=\Delta \boldsymbol{\Phi}^{\perp}+\boldsymbol{A} \boldsymbol{\Phi}-\boldsymbol{\Phi} \boldsymbol{A},
$$

where the $r \times r$ matrix $\Delta \boldsymbol{\Phi}^{\perp}$ is defined as

$$
\Delta \boldsymbol{\Phi}^{\perp}=-\boldsymbol{W}_{\downarrow}^{\dagger} \Delta \boldsymbol{W}_{\downarrow}^{\perp} \boldsymbol{\Phi}+\boldsymbol{\Phi} \boldsymbol{W}_{\uparrow}^{\dagger} \Delta \boldsymbol{W}_{\uparrow}^{\perp} .
$$

${ }^{16}$ To compute $\nabla \mathcal{J}(\boldsymbol{U})$, the following derivation rule has to be applied: if $\boldsymbol{M}$ is a constant $n \times r$ matrix, $\boldsymbol{\nabla}$ trace $\left(\Re\left(\boldsymbol{U}^{H} \boldsymbol{M}\right)\right)=\boldsymbol{M}$. As a consequence, if $\boldsymbol{C}$ is a constant $n \times n$ hermitian matrix, $\boldsymbol{\nabla}$ trace $\left(\boldsymbol{U}^{H} \boldsymbol{C U}\right)=$ $2 \boldsymbol{C U}$.
Equations (34) to (36) are obtained by substituting equations (27) to (29) into the first order expansion of equation (33) which defines $\Phi(\varepsilon)$.

In the following step, the estimated poles are defined as the eigenvalues of the perturbed spectral matrix $\Phi(\varepsilon)$. In order to compute their first order expansion, we first need to introduce the matrix $\boldsymbol{J}(\varepsilon) \triangleq \boldsymbol{G}^{-1} \boldsymbol{\Phi}(\varepsilon) \boldsymbol{G}$, which is similar to $\boldsymbol{\Phi}(\varepsilon)$; thus the estimated poles can also be viewed as the eigenvalues of $\boldsymbol{J}(\varepsilon)$. Note however that $\boldsymbol{J}(\varepsilon)$ is generally no longer a Jordan matrix when $\varepsilon>0$. The following corollary provides the first order expansion of this matrix. Let us define the vectors $\boldsymbol{v}_{\uparrow}$ and $\boldsymbol{v}_{\downarrow}$ of same dimension $r$ as the conjugate transpose of the first and last row of the matrix $\boldsymbol{V}^{n}$ respectively, and consider the $r \times r$ positive definite matrix $\boldsymbol{Z}=\boldsymbol{V}^{n H} \boldsymbol{V}^{n}$.

Corollary 11. Let $\boldsymbol{J}(\varepsilon)=\boldsymbol{G}^{-1} \boldsymbol{\Phi}(\varepsilon) \boldsymbol{G}$. The function $\varepsilon \mapsto \boldsymbol{J}(\varepsilon)$ is $\mathcal{C}^{\infty}$ in a neighborhood of $\varepsilon=0$, and admits the expansion

$$
\boldsymbol{J}(\varepsilon)=\boldsymbol{J}+\varepsilon \Delta \boldsymbol{J}+O\left(\varepsilon^{2}\right)
$$

where the first order perturbation $\Delta \boldsymbol{J}=\boldsymbol{G}^{-1} \Delta \boldsymbol{\Phi} G$ can be written in the following form (where $\boldsymbol{A}^{\prime}=\boldsymbol{G}^{-1} \boldsymbol{A} \boldsymbol{G}$ ):

$$
\Delta J=\Delta J^{\perp}+A^{\prime} J-J A^{\prime}
$$

Moreover, the matrix $\Delta \boldsymbol{J}^{\perp}=\boldsymbol{G}^{-1} \Delta \boldsymbol{\Phi}^{\perp} \boldsymbol{G}$ has rank 2:

$$
\Delta \boldsymbol{J}^{\perp}=\boldsymbol{v}_{\downarrow}^{\prime} \boldsymbol{e}_{\downarrow}{ }^{H} \Delta \boldsymbol{S} \boldsymbol{V}^{l^{\dagger T}} \boldsymbol{D}^{-1} \boldsymbol{J}-\boldsymbol{J} \boldsymbol{v}_{\uparrow}^{\prime} \boldsymbol{e}_{\uparrow}^{H} \Delta \boldsymbol{S} \boldsymbol{V}^{l^{\dagger T}} \boldsymbol{D}^{-1}
$$

where the $l \times r$ Pascal-Vandermonde matrix $\boldsymbol{V}^{l}$ and the $r \times r$ block-diagonal matrix $\boldsymbol{D}$ were introduced in proposition 1 , the $r$ dimensional vectors $\boldsymbol{v}_{\downarrow}^{\prime}$ and $\boldsymbol{v}_{\uparrow}^{\prime}$ are defined as

$$
\begin{aligned}
\boldsymbol{v}_{\downarrow}^{\prime} & =\frac{\boldsymbol{Z}^{-1} \boldsymbol{v}_{\downarrow}}{1-\boldsymbol{v}_{\downarrow}^{H} \boldsymbol{Z}^{-1} \boldsymbol{v}_{\downarrow}} \\
\boldsymbol{v}_{\uparrow}^{\prime} & =\frac{\boldsymbol{Z}^{-1} \boldsymbol{v}_{\uparrow}}{1-\boldsymbol{v}_{\uparrow}^{H} \boldsymbol{Z}^{-1} \boldsymbol{v}_{\uparrow}},
\end{aligned}
$$

and the n-dimensional vectors $\boldsymbol{e}_{\downarrow}$ and $\boldsymbol{e}_{\uparrow}$ are defined as

$$
\begin{aligned}
& \boldsymbol{e}_{\downarrow}=[0 \ldots 0,1]^{T}-\boldsymbol{V}^{n} \boldsymbol{Z}^{-1} \boldsymbol{v}_{\downarrow} \\
& \boldsymbol{e}_{\uparrow}=[1,0 \ldots 0]^{T}-\boldsymbol{V}^{n} \boldsymbol{Z}^{-1} \boldsymbol{v}_{\uparrow} .
\end{aligned}
$$

Corollary 11 is derived from proposition 10 by means of the basis change (7).

3) Perturbation of the poles: Finally, we focus in this section on the perturbation of the poles. Theoretically, they are obtained by computing the Jordan form of the spectral matrix $\boldsymbol{\Phi}$. In practice, contrary to $\boldsymbol{\Phi}$, the perturbed spectral matrix does not have multiple eigenvalues: multiple poles are scattered into several single eigenvalues.

More precisely, it was shown in [2] that if $M_{k}>1$ :

- the first order perturbation of the pole $z_{k}$ is homogeneous and isotropic, so that the $M_{k}$ perturbed eigenvalues $z_{k}^{(m)}(\varepsilon)$ form the vertices of a regular polynomial of order $M_{k}$ in the complex plan;

- the perturbation of the scattered eigenvalues is of order $\varepsilon^{\frac{1}{M_{k}}}$, which suggests that multiple poles are more sensitive to perturbations than single poles.

In fact it is possible to overcome this problem by no longer considering the eigenvalues $z_{k}^{(m)}(\varepsilon)$ as $M_{k}$ distinct estimators of the same pole $z_{k}$, but rather by forming a single estimator of this pole by averaging the $z_{k}^{(m)}(\varepsilon)$. The following proposition shows that the arithmetic mean $z_{k}(\varepsilon)$ of the scattered eigenvalues admits a series expansion. 
Proposition 12 (Perturbation of the poles). Let $z_{k}(\varepsilon)=$ $\frac{1}{M_{k}} \sum_{m=0}^{M_{k}-1} z_{k}^{(m)}(\varepsilon) \forall k \in\{0 \ldots K-1\}$. Then the function $\varepsilon \mapsto z_{k}(\varepsilon)$ is $\mathcal{C}^{\infty}$ and admits the first order expansion

$$
z_{k}(\varepsilon)=z_{k}+\varepsilon \Delta z_{k}+O\left(\varepsilon^{2}\right)
$$

where $\Delta z_{k}$ is the complex number

$$
\Delta z_{k}=\frac{1}{M_{k}} \operatorname{trace}\left(\Delta \boldsymbol{J}_{k}^{\perp}\right) .
$$

Here, $\Delta \boldsymbol{J}_{k}^{\perp}$ is the sub-block of dimension $M_{k} \times M_{k}$ extracted from the matrix $\Delta \boldsymbol{J}^{\perp}$, which is associated to $z_{k}{ }^{17}$. The complex number $\Delta z_{k}$ can also be written in the form

$$
\Delta z_{k}=\frac{1}{M_{k} \alpha_{k}^{\left(M_{k}-1\right)}}\left(\boldsymbol{e}_{\downarrow}^{H} \Delta \boldsymbol{S} \boldsymbol{f}_{\downarrow k}-\boldsymbol{e}_{\uparrow}^{H} \Delta \boldsymbol{S} \boldsymbol{f}_{\uparrow k}\right) .
$$

The l-dimensional vectors $\boldsymbol{f}_{\downarrow k}$ and $\boldsymbol{f}_{\uparrow k}$ are defined ${ }^{18}$ as

$$
\left\{\begin{array}{l}
\boldsymbol{f}_{\downarrow k}=\alpha_{k}^{\left(M_{k}-1\right)} \boldsymbol{V}^{l^{\dagger T}} \boldsymbol{D}^{-1} \boldsymbol{J} \boldsymbol{v}_{\downarrow k}^{\prime} \\
\boldsymbol{f}_{\uparrow k}=\alpha_{k}^{\left(M_{k}-1\right)} \boldsymbol{V}^{l^{\dagger T}} \boldsymbol{D}^{-1} \boldsymbol{J} \boldsymbol{v}_{\uparrow k}^{\prime},
\end{array}\right.
$$

where $\boldsymbol{v}_{\downarrow k}^{\prime}$ and $\boldsymbol{v}_{\uparrow k}^{\prime}$ are the r-dimensional vectors whose coefficients are equal to those of $\boldsymbol{v}_{\downarrow}^{\prime}$ and $\boldsymbol{v}_{\uparrow}^{\prime}$ inside the $k^{\text {th }}$ sub-block ${ }^{19}$, and zero outside this sub-block.

Equation (42) is derived by substituting equation (39) into equation (41). It can be noticed that the antihermitian matrix $\boldsymbol{A}$, introduced in proposition 9, is no longer involved in proposition 12 . We can conclude that the performance of the generalized ESPRIT algorithm is not sensitive to the particular choice of the orthonormal basis $\boldsymbol{W}(\varepsilon)$ (at the first order)

Since the matrix $\Delta S$ is Hankel and contains the samples of the PACE signal, the right member of equation (42) contains linear combinations of $\Delta s(\tau)$ for $\tau \in\{t-l+1 \ldots t+n-1\}$. Therefore equation (42) can also be written as a scalar product:

$$
\Delta z_{k}=\frac{1}{M_{k} \alpha_{k}^{\left(M_{k}-1\right)}} \boldsymbol{u}_{k}{ }^{H} \Delta s
$$

where for all $\tau \in[0, \ldots, n+l-2]$, the coefficient of index $\tau$ in $\boldsymbol{u}_{k}$ is $^{20}$

$u_{k}(\tau)=\sum_{v=0}^{n-1}\left(e_{\downarrow}(v) f_{\downarrow k}^{*}(\tau-v)-e_{\uparrow}(v) f_{\uparrow k}^{*}(\tau-v)\right) \mathbf{1}_{\{\tau-l<v \leq \tau\}}$.

Equation (45) involves two convolution products, which are due to the Hankel matrix / vector products in equation (42).

Finally, proposition 3 in section III shows how the perturbation of the poles affects the frequencies and damping factors.

\section{B. Perturbation of the amplitudes and phases}

Below, we successively derive the following first order expansions:

- $\boldsymbol{V}^{N}(\varepsilon)=\boldsymbol{V}^{N}+\varepsilon \Delta \boldsymbol{V}^{N}+O\left(\varepsilon^{2}\right)$, where the expression of $\Delta \boldsymbol{V}^{N}$ is a linear function of the $\Delta z_{k}$ 's (lemma 13);

- $\boldsymbol{\alpha}(\varepsilon)=\boldsymbol{\alpha}+\varepsilon \Delta \boldsymbol{\alpha}+O\left(\varepsilon^{2}\right)$, where the expression of $\Delta \boldsymbol{\alpha}$ is a linear function of $\Delta s$ (proposition 14); - $\left\{\begin{array}{c}a_{k}(\varepsilon)=a_{k}+\varepsilon \Delta a_{k}+O\left(\varepsilon^{2}\right) \\ \phi_{k}(\varepsilon)=\phi_{k}+\varepsilon \Delta \phi_{k}+O\left(\varepsilon^{2}\right) \\ \Delta a_{k} \text { and } \Delta \phi_{k} \text { are functions of } \Delta \boldsymbol{\alpha} \text { (corollary 4). }\end{array}\right.$

${ }^{17}$ This corresponds to the rows and columns of indices $\sum_{k^{\prime}=0}^{k-1} M_{k^{\prime}}$ to $\sum_{k^{\prime}=0}^{k} M_{k^{\prime}}-1$.

${ }^{18}$ It can be verified that if $M_{k}=1$, the vectors $\boldsymbol{f}_{\downarrow k}$ and $\boldsymbol{f}_{\uparrow k}$ do not depend on any complex amplitude.

${ }^{19}$ This corresponds to the coefficients of indices $\sum_{k^{\prime}=0}^{k-1} M_{k^{\prime}}$ to $\sum_{k^{\prime}=0}^{k} M_{k^{\prime}}-1$.

${ }^{20}$ Here the function $\mathbf{1}_{(.)}$is one if its argument is true and zero otherwise.

\section{1) Perturbation of the Pascal-Vandermonde matrix:}

Lemma 13 (Perturbation of the Pascal-Vandermonde matrix). Let $V^{N}(\varepsilon)$ be the $N \times r$ Pascal-Vandermonde matrix associated to the estimated poles $\left\{z_{0}(\varepsilon), \ldots, z_{K-1}(\varepsilon)\right\}$ defined in proposition 12 . Then the function $\varepsilon \mapsto \boldsymbol{V}^{N}(\varepsilon)$ is $\mathcal{C}^{\infty}$ in the neighborhood of $\varepsilon=0$, and admits the first order expansion:

$$
\boldsymbol{V}^{N}(\varepsilon)=\boldsymbol{V}^{N}+\varepsilon \Delta \boldsymbol{V}^{N}+O\left(\varepsilon^{2}\right)
$$

where the matrix $\Delta \boldsymbol{V}^{N}$ can be written in the form

$$
\Delta \boldsymbol{V}^{N}=\overline{\boldsymbol{V}}^{N} \Delta \boldsymbol{Z}
$$

where $\overline{\boldsymbol{V}}^{N}$ is the $N \times(r+K)$ Pascal-Vandermonde matrix obtained by concatenating the generalized Pascal matrices $\bar{C}_{k}^{N}=$ $C_{M_{k}+1}^{N}\left(z_{k}\right)$ of dimension $N \times\left(M_{k}+1\right)$, and

$$
\Delta \boldsymbol{Z}=\operatorname{diag}\left(\Delta \boldsymbol{Z}_{0}, \ldots, \Delta \boldsymbol{Z}_{K-1}\right)
$$

is a $(r+K) \times r$ matrix whose diagonal blocks

$$
\Delta \boldsymbol{Z}_{k}=\Delta z_{k} \times\left[\begin{array}{c}
\mathbf{0}_{\left(1 \times M_{k}\right)} \\
\operatorname{diag}\left(1,2, \ldots, M_{k}\right)
\end{array}\right]
$$

have dimension $\left(M_{k}+1\right) \times M_{k}$.

Lemma 13 is proved by substituting the first order expansion of the poles (40) into the expression of the Pascal-Vandermonde matrix, and by extracting the terms of order 1 .

2) Perturbation of the amplitudes and phases: The perturbation of the complex-valued amplitudes can be derived from lemma 13. Let

$$
s=[s(t-l+1), \ldots, s(t+n-1)]^{T}
$$

be the $N$-dimensional vector containing the samples of the PACE signal. For all $\varepsilon \in \mathbb{R}$, the $N$-dimensional observed vector is $s(\varepsilon)=$ $s+\varepsilon \Delta s$.

Proposition 14 (Perturbation of the complex-valued amplitudes). The perturbed LS estimate defined in equation (9) can be written in the form

$$
\boldsymbol{\alpha}(\varepsilon)=\boldsymbol{V}^{N}(\varepsilon)^{\dagger} \boldsymbol{s}(\varepsilon)
$$

Then the function $\varepsilon \mapsto \boldsymbol{\alpha}(\varepsilon)$ is $\mathcal{C}^{\infty}$ in the neighborhood of $\varepsilon=0$, and admits the first order expansion:

$$
\alpha(\varepsilon)=\alpha+\varepsilon \Delta \alpha+O\left(\varepsilon^{2}\right)
$$

The r-dimensional vector $\Delta \boldsymbol{\alpha}$ satisfies

$$
\Delta \boldsymbol{\alpha}=\boldsymbol{B}^{H} \Delta s
$$

where the $r \times N$ matrix

$$
\boldsymbol{B}^{H}=\boldsymbol{V}^{N^{\dagger}}\left(\boldsymbol{I}_{N}-\overline{\boldsymbol{V}}^{N}\left[\begin{array}{c}
\boldsymbol{B}_{0} \\
\vdots \\
\boldsymbol{B}_{K-1}
\end{array}\right]\right)
$$

involves the $\left(M_{k}+1\right) \times N$ matrices $\boldsymbol{B}_{k}$, of rank 1:

$$
\boldsymbol{B}_{k}=\left[0, \frac{1}{M_{k}} \frac{\alpha_{k}^{(0)}}{\alpha_{k}^{\left(M_{k}-1\right)}}, \ldots, \frac{M_{k}-1}{M_{k}} \frac{\alpha_{k}^{\left(M_{k}-2\right)}}{\alpha_{k}^{\left(M_{k}-1\right)}}, 1\right]^{T} \boldsymbol{u}_{k}^{H} .
$$

Equations (51) to (54) are obtained by substituting equations (46) to (49) into the first order expansion of equation (50).

Finally, proposition 4 in section III shows how the perturbation of the complex-valued amplitudes influences the real-valued amplitudes and phases. The vector $\boldsymbol{b}_{k}^{(m)}$ is the column of $\boldsymbol{B}$ associated to the pole $z_{k}$ at index $m$, i.e. the column of index $m+\sum_{k^{\prime}=0}^{k-1} M_{k^{\prime}}$. The derivation of equation (14) from equation (52) is straightforward. 


\section{REFERENCES}

[1] R. Roy, A. Paulraj, and T. Kailath, "ESPRIT-A subspace rotation approach to estimation of parameters of cisoids in noise," IEEE Trans. Acoust., Speech, Signal Processing, vol. 34, no. 5, pp. 1340-1342, Oct. 1986.

[2] R. Badeau, B. David, and G. Richard, "High resolution spectral analysis of mixtures of complex exponentials modulated by polynomials," IEEE Trans. Signal Processing, vol. 54, no. 4, pp. 1341-1350, Apr. 2006.

[3] R. A. Serway and J. W. Jewett, Physics for Scientists and Engineers. Brooks/Cole, 2003.

[4] B. Fischer and A. Medvedev, " $\mathbf{L}^{2}$ Time Delay Estimation by Means of Laguerre Functions," in Proceedings of the American Control Conference, San Diego, California, USA, June 1999.

[5] A. M. Sabatini, "Correlation Receivers Using Laguerre Filter Banks for Modelling Narrowband Ultrasonic Echoes and Estimating Their Timeof-Flights," IEEE Trans. Ultrason., Ferroelect., Freq. Contr., vol. 44, no. 6, pp. 1253-1263, Nov. 1997.

[6] O. V. Ivanova, L. Marcu, and M. C. K. Khoo, "A Nonparametric Method for Analysis of Fluorescence Emission in Combined Time and Wavelength Dimensions," Annals of Biomedical Engineering, vol. 33, no. 4, pp. 531-544, Apr. 2005.

[7] T. W. Hänsch, A. L. Schawlow, and G. W. Series, "The Spectrum of Atomic Hydrogen," Sci. Amer., vol. 240, no. 94, pp. 531-544, Mar. 1979.

[8] P. W. Milonni and J. H. Eberly, Lasers, ser. Wiley Series in Pure and Applied Optics. New York, USA: Wiley-Interscience, 1988.

[9] D. Filipovic, "Exponential-polynomial families and the term structure of interest rates," Bernoulli, vol. 6, no. 6, pp. 1081-1107, 2000.

[10] V. Slivinskas, M. Radavicius, and V. Simonyte, "Cramér-rao bound for the estimates of frequencies and damping factors of quasipolynomials in noise," Report UPTEC 92022R, Institute of Technology, Uppsala University, Sweden, Tech. Rep., Feb. 1992.

[11] S. M. Kay, Fundamentals of Statistical Signal Processing: Estimation Theory. Englewood Cliffs, NJ, USA: Prentice-Hall, 1993.

[12] R. Badeau, B. David, and G. Richard, "Cramér-Rao bounds for multiple poles and coefficients of quasipolynomials in colored noise," submitted for publication.

[13] A. Kot, S. Parthasarathy, D. Tufts, and R. Vaccaro, "The statistical performance of state-variable balancing and Prony's method in parameter estimation," in Proc. of ICASSP'87, vol. 12, Apr. 1987, pp. 1549-1552.

[14] P. Stoica and A. Nehorai, "Study of the statistical performance of the Pisarenko harmonic decomposition method," IEE Proceedings Radar and Signal Processing, vol. 135, no. 2, pp. 161-168, Apr. 1988.

[15] G. M. Riche de Prony, "Essai expérimental et analytique sur les lois de la dilatabilité de fluides élastiques et sur celles de la force expansive de la vapeur de l'eau et de la vapeur de l'alcool à différentes températures," Journal de l'école polytechnique, vol. 1, no. 22, pp. 24-76, 1795, in French.

[16] V. F. Pisarenko, "The retrieval of harmonics from a covariance function," Geophysical J. Royal Astron. Soc., vol. 33, pp. 347-366, 1973.

[17] R. Kumaresan and D. W. Tufts, "Estimating the parameters of exponentially damped sinusoids and pole-zero modeling in noise," IEEE Trans. Acoust., Speech, Signal Processing, vol. 30, no. 6, pp. 833-840, Dec. 1982.

[18] R. O. Schmidt, "Multiple emitter location and signal parameter estimation," IEEE Trans. Antennas Propagat., vol. 34, no. 3, pp. 276-280, Mar. 1986.

[19] Y. Hua and T. K. Sarkar, "Matrix pencil method for estimating parameters of exponentially damped/undamped sinusoids in noise," IEEE Trans. Acoust., Speech, Signal Processing, vol. 38, no. 5, pp. 814-824, May 1990.

[20] B. Porat and B. Friedlander, "On the accuracy of the Kumaresan-Tufts method for estimating complex damped exponentials," IEEE Trans. Acoust., Speech, Signal Processing, vol. 35, no. 2, pp. 231-235, Feb. 1987.

[21] Y. Hua and T. K. Sarkar, "Perturbation analysis of TK method for harmonic retrieval problems," IEEE Trans. Acoust., Speech, Signal Processing, vol. 36, pp. 228-240, Feb. 1988.

[22] P. Stoica and T. Söderström, "Statistical Analysis of MUSIC and Subspace Rotation Estimates of Sinusoidal Frequencies," IEEE Trans. Signal Processing, vol. 39, pp. 1836-1847, Aug. 1991.

[23] A. Eriksson, P. Stoica, and T. Soderstrom, "Second-order properties of MUSIC and ESPRIT estimates of sinusoidal frequencies in high SNR scenarios," IEE Proceedings on Radar, Sonar and Navigation, vol. 140, no. 4, pp. 266-272, Aug. 1993.
[24] Y. Hua and T. K. Sarkar, "On SVD for estimating generalized eigenvalues of singular matrix pencil in noise," IEEE Trans. Signal Processing, vol. 39, no. 4, pp. 892-900, Apr. 1991.

[25] S. Roman, The Umbral Calculus. New York: Academic Press, 1984, $\S 1.2$ : The Lower Factorial Polynomial.

[26] R. L. Graham, D. E. Knuth, and O. Patashnik, Concrete Mathematics: A Foundation for Computer Science, 2nd ed. Reading, MA: AddisonWesley, 1994.

[27] T. Kailath, Linear systems. Englewood Cliffs, New Jersey, USA: Prenctice-Hall, 1980.

[28] R. A. Horn and C. R. Johnson, Matrix analysis. Cambridge University Press, 1985.

[29] R. Badeau, G. Richard, and B. David, "Sliding window adaptive SVD algorithms," IEEE Trans. Signal Processing, vol. 52, no. 1, pp. 1-10, Jan. 2004.

[30] R. Badeau, B. David, and G. Richard, "Fast Approximated Power Iteration Subspace Tracking," IEEE Trans. Signal Processing, vol. 53, no. 8, pp. 2931-2941, Aug. 2005.

[31] _ - "Yet Another Subspace Tracker," in Proc. of ICASSP'05, vol. 4. IEEE, Mar. 2005, pp. 329-332.

[32] H. Zeiger and A. McEwen, "Approximate linear realizations of given dimension via Ho's algorithm," IEEE Trans. Automat. Contr., vol. 19, no. 2, p. 153 , Apr. 1974.

[33] R. Badeau, B. David, and G. Richard, "A new perturbation analysis for signal enumeration in rotational invariance techniques," IEEE Trans. Signal Processing, vol. 54, no. 2, pp. 450-458, Feb. 2006.

[34] Z. Zeng, "On ill-conditioned eigenvalues, multiple roots of polynomials, and their accurate computations," MSRI Preprint No. 1998-048, Northeastern Illinois University, Chicago, USA, Tech. Rep., 1998.

[35] M. Wax and T. Kailath, "Detection of signals by information theoretic criteria," IEEE Trans. Acoust., Speech, Signal Processing, vol. 33, no. 2, pp. 387-392, Apr. 1985.

[36] K. Abed-Meraim, P. Loubaton, and E. Moulines, "A Subspace Algorithm for Certain Blind Identification Problems," IEEE Trans. Inform. Theory, vol. 43, no. 2, pp. 499-511, Mar. 1997.

[37] B. Yang, "Projection Approximation Subspace Tracking," IEEE Trans. Signal Processing, vol. 44, no. 1, pp. 95-107, Jan. 1995. 